\title{
Linfoma tipo Burkitt, un inusual subtipo de linfoma testicular no Hodgkin en el adulto: reporte de un caso y revisión de la literatura
}

\section{Burkitts Lymphoma, an Unusual Subtype of Non- Hodgkin Testicular Lymphoma in the Adult: Repor of a Case and Review of the Literatura}

\author{
Diana María Cerquera Cleves ${ }^{1}$ Wilfredo Donoso Donoso ${ }^{2}$
}

\footnotetext{
${ }^{1}$ Residente de segundo año de Urología, Universidad Nacional de Colombia, Bogotá D.C, Colombia

${ }^{2}$ Urólogo, Jefe del Servicio de Urología, Universidad Nacional de Colombia, Bogotá D.C, Colombia
}

\begin{abstract}
Address for correspondence Dra. Diana María Cerquera Cleves, MD, Universidad Nacional de Colombia, Transversal 58 \# 108-36, Bogotá, Colombia (e-mail: dianitamcc19@hotmail.com).
\end{abstract}

Urol Colomb 2019;28:9-14.

\section{Resumen \\ Palabras Claves \\ - linfoma no Hodgkin \\ - testículo \\ - Burkitt \\ - ecografía doppler \\ - orquiectomía \\ - metástasis}

Objetivo Realizar el reporte de un caso de linfoma testicular no Hodgkin tipo Burkitt, presentando una revisión de la literatura y documentando las variaciones histológicas típicas, los métodos diagnósticos, el impacto del manejo inicial y el abordaje de las metástasis e infiltraciones tumorales en el pronóstico de esa patología oncológica.

Metodología Se describe el caso de un hombre de 36 años en quien se diagnosticó un linfoma testicular tipo Burkitt, durante el estudio de un síndrome proliferativo, que inició con signos y síntomas neurológicos conllevando a la extensión del estudio y demostrando múltiples lesiones metastásicas asociadas a la infiltración del sistema nervioso central. El tratamiento incluye múltiples modalidades entre las que se encuentra la quimioterapia, radioterapia local, profilaxis intratecal entre otras; sin embargo, el pilar del manejo del compromiso testicular sigue siendo el quirúrgico.

Conclusión El linfoma testicular no hodking es una patología rara, siendo aún más infrecuente el subtipo Burkitt, a pesar del pobre pronóstico, el diagnóstico oportuno y el desarrollo de nuevas terapias aplicadas en combinación, han permitido mejorar la sobrevida y disminuir el riesgo de recaída y progresión.

\footnotetext{
Abstract

Purpose To report a case of Burkitt-type non-Hodgkin's lymphoma, presenting a review of the literature and documenting the typical histological variations, diagnostic methods, and the impact of initial management and treatment of metastases and tumor infiltrations in the prognosis of this oncological pathology.
}

received January 7, 2018 accepted March 22, 2018 published online July 10, 2018
DOI https://doi.org/ 10.1055/s-0038-1648240. ISSN 0120-789X. eISSN 2027-0119.
Copyright ( 2019, Sociedad Colombiana License terms de Urología. Publicado por Thieme Revinter Publicações Ltda., Rio de Janeiro, Brazil. Todos los derechos reservados.

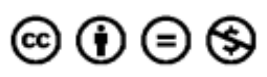




\author{
Keywords \\ - non-Hodgkin's \\ lymphoma \\ - testis \\ - Burkitt \\ - doppler ultrasound \\ - orchiectomy \\ - metastasis
}

Methodology We describe the case of a 36-year-old man diagnosed with Burkitt-type testicular lymphoma during the study of a proliferative syndrome, which began with neurological signs and symptoms, evidencing multiple metastatic lesions with infiltration the central nervous system. Treatment includes multiple modalities including chemotherapy, local radiotherapy, intrathecal prophylaxis among others; However, The pillar of treatment is the surgical procedure.

Conclusion Non-hodking testicular lymphoma is a rare condition, and the Burkitt subtype is even rarer. Despite the poor prognosis, early diagnosis and the development of new therapies in combination, have improved survival and reduced the risk of relapse and progression.

\section{Introducción}

El linfoma testicular representa solo entre el $1 \%$ y el $2 \%$ de los linfomas no Hodgkin, y del 1\% al 7\% de los tumores testiculares, encontrando su mayor incidencia en adultos mayores de 50 años. El subtipo más frecuente es el difuso de células B gigantes, sin embargo, existen otros subtipos cuyos casos han sido reportados como el linfoma de Burkitt. Ese tipo de linfomas, tiene tendencia a la diseminación extranodal, permitiendo de esa forma, que existan una gran variedad de manejos sistémicos para lograr un adecuado control oncológico. ${ }^{1}$

Los linfoma de Burkitt son subtipos del linfoma no Hodgkin no muy comunes pero endémicos en regiones africanas, evidenciándose una asociación importante con la infección con el virus de Epstein Barr; por otra parte, la forma esporádica solo tiene asociación en el $15 \%$ de los casos con ese virus. Es un subtipo agresivo y requiere de estudios imagenológicos de urgencia para descartar invasión visceral asociada. ${ }^{2}$

El compromiso extranodal puede comprometer diferentes órganos como el estómago, pulmón, pleura, cerebro (6-16\%), intestino delgado, piel (6-35\%), e inclusive se conocen reportes de compromiso del anillo de Waldeyer (5\%) y el testículo contralateral. ${ }^{3}$ Los factores de riesgo que contribuyen al desarrollo de linfoma no Hodgkin, se han visto a su vez relacionados con la aparición de enfermedad extranodal, por ese motivo, su incidencia es variable, sin embargo, hay reportes de entre el 20\% al 34\% dependiendo de los criterios adoptados. ${ }^{4}$

Con respecto al manejo del linfoma testicular no Hodgkin, por muchos años consistía en sesiones de radioterapia para la enfermedad localizada y quimioterapia para el compromiso sistémico, sin embargo, debido al pobre pronóstico y alto riesgo de recaída, hoy en día diferentes series y estudios retrospectivos y algunos prospectivos, han indicado el uso del manejo combinado con orquiectomía radical, radioterapia regional y quimioterapia dual inclusive en estadios tempranos con resultados diversos. ${ }^{5}$

\section{Caso Clínico}

Hombre de 36 años que ingresa por cuadro de una semana de evolución posterior a politrauma secundario a accidente de tránsito, de dolor en flanco y fosa iliaca izquierda, asociado a edema inguino-escrotal y de miembros inferiores, diplopía, cefalea y hemiparesia de miembro inferior izquierdo $\mathrm{y}$ maxilar inferior, al interrogatorio asocia diaforesis nocturna de larga data.

Además de presentar hipertensión arterial y ser fumador pesado, se documenta historia familiar de neoplasia gástrica en hermana y leucemia en hermano con fallecimiento antes de la $4^{\text {ta }}$ década de la vida, sin estudio genético previo.

$\mathrm{Al}$ ingreso se documenta en ecografía abdominal extrainstitucional adenomegalias inguinales izquierdas, y lesiones hepáticas que podrían corresponder a metástasis, por ese motivo se realiza tomografía axial computarizada (TAC) de abdomen simple y contrastado, y en ella se confirman múltiples adenomegalias retroperitoneales e inguinales izquierdas y conglomerados adyacentes a los vasos iliacos izquierdos, visualizando al igual lesiones en el parénquima hepático mayores de $2 \mathrm{cms}$ con adenomegalias de implantación peritoneal y en región perirenal derecha sin hidronefrosis ni uropatía obstructiva asociada (-Fig. 1). Esas lesiones tumorales generaban efecto compresivo vascular en la vena iliaca común y femoral izquierda, descartándose la presencia de trombosis venosa profunda con ecografía doppler. Para el inicio del estudio histopatológico realizan biopsia trucut de lesión focal hepática izquierda.

Ante la persistencia del deterioro neurológico secundario a un síndrome por parálisis del VI par, se realiza resonancia magnética (RM) cerebral contrastada que documenta masa paraselar izquierda con leve desplazamiento del tallo hipofisiario hacia la derecha de posible aspecto metastásico.

Durante el estudio de neoplasia primaria realizan TAC de tórax contrastado con evidencia de adenomegalia pre vascular y nódulos de tejido blando y contornos mal definidos en ambos campos pulmonares, sin poder descartarse compromiso neoplásico secundario. El paciente presenta signos de deterioro respiratorio, confirmándose trombo embolismo pulmonar e iniciando anticoagulación plena con heparina de bajo peso molecular.

Por evidencia de aumento del contenido escrotal con edema de pared que dificulta la palpación testicular, se realiza ecografía doppler testicular con documentación de dos lesiones hipoecoicas en polo inferior de testículo izquierdo (-Fig. 2). Bajo esos hallazgos ecográficos, y en el contexto del paciente con sospecha de neoplasia testicular, 

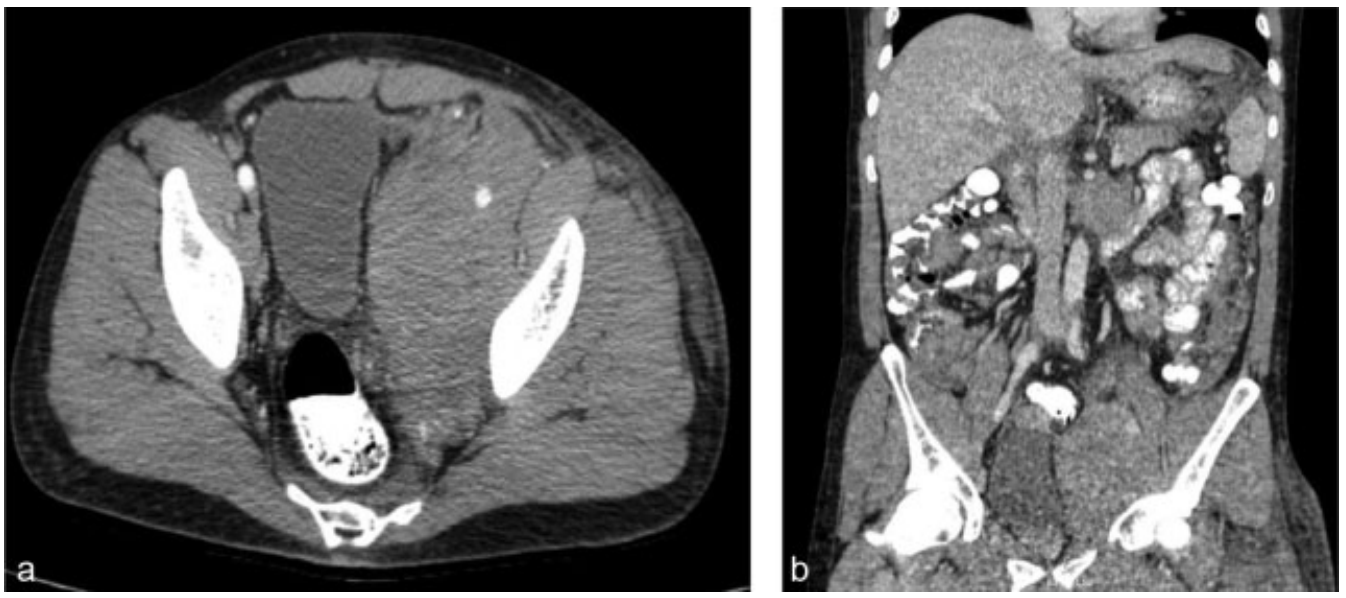

Fig. 1 (a-b) TAC de Abdomen simple y contrastado. Adenomegalias retroperitoneales inferior a la arteria mesentérica superior, conglomerados adyacentes a vasos iliacos izquierdos con diámetro mayor de $14 \mathrm{~cm}$ Adenomegalias inguinales

se solicitan marcadores tumorales negativos (BHCG $0.1019 \mathrm{y}$ AFP 1.53), con marcador de carga tumoral elevado (LDH: 3403/232); posteriormente se realiza orquiectomía radical izquierda con visualización de testículo macroscópicamente normal, con induración de $1 \mathrm{~cm}$ en polo inferior.

A pesar del manejo quirúrgico instaurado, el paciente presenta progresión de paraparesia de miembros inferiores, sin nivel sensitivo asociado, que lleva a la realización de gamagrafia ósea negativa para metástasis y RMN de columna dorsal ( - Fig. 3), con lesión focal de aspecto lítico y neoplásico de T6 a T8 con compresión a nivel de T7 y T8. Se realiza manejo quirúrgico para compresión medular con laminectomía de T6-T7 y extracción de lesión epidural compresiva.

Se instaura un deterioro progresivo en el posoperatorio, con requerimiento de soporte vasopresor y de ventilación mecánica por choque multifactorial con síndrome febril persistente a pesar de cultivos sin aislamiento de microorganismo. Patología de biopsia hepática y espécimen testicular reporta hallazgos
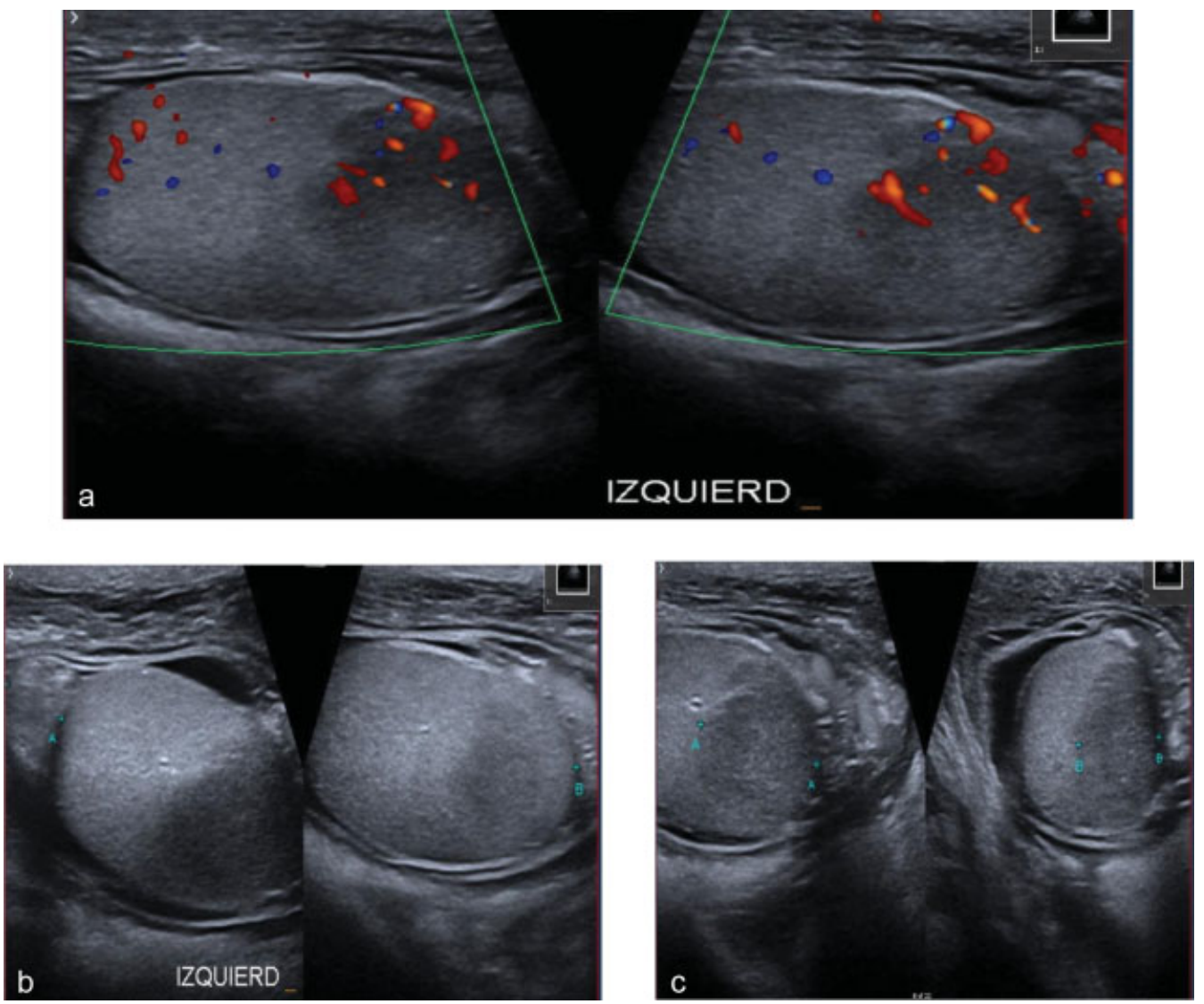

Fig. 2 (a-c) Ecografía doppler testicular. Testículo izquierdo con dos lesiones hipoecoicas de aspecto sólido y contornos parcialmente definidos; una en tercio medio de $41 \times 27 \times 29 \mathrm{~mm}$ y otra en el inferior de $39 \times 27 \times 27 \mathrm{~mm}$. El análisis doppler demuestra flujo adecuado intratesticular y epididimario de manera bilateral, con ligera hipervascularización de las estructuras en el lado izquierdo. 

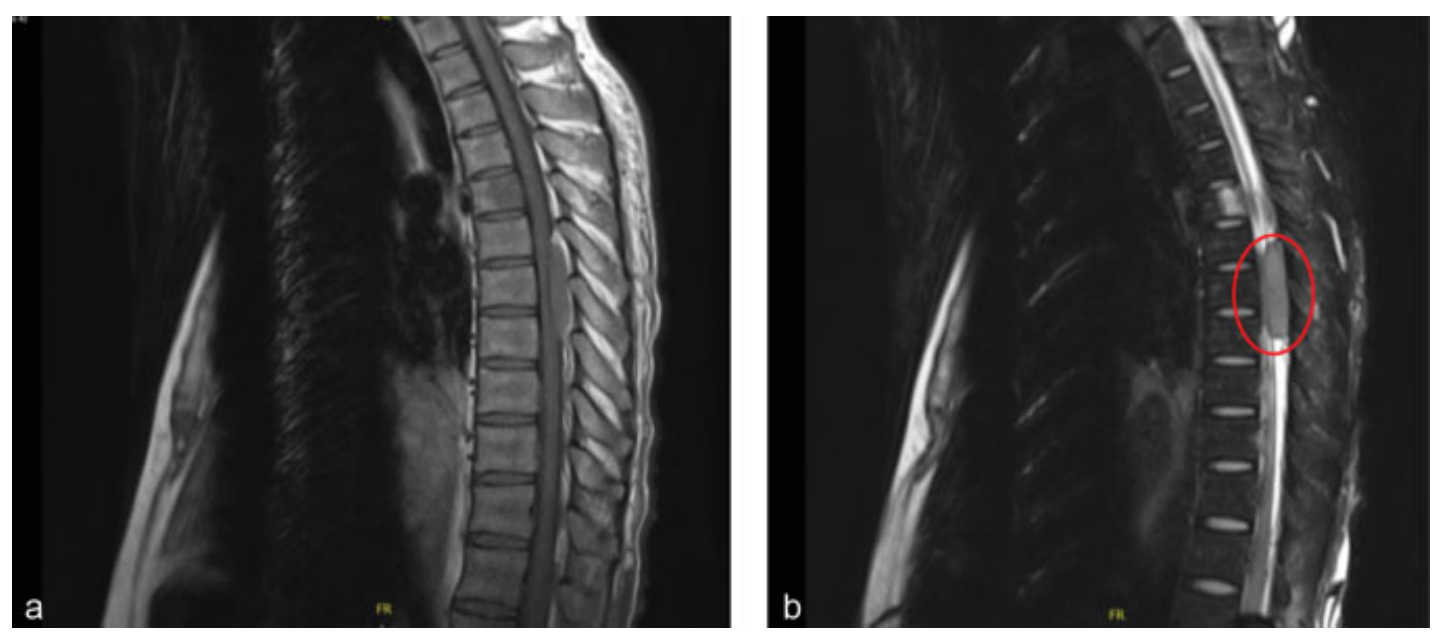

Fig. 3 (a-b) RM Lumbar. Masa con localización epidural posterior entre T6 y T8, obliterando el canal medular, y extendiéndose a través del agujero de conjunción izquierdo T2-T8.

sugestivos de neoplasia linfoproliferativa compatible con Linfoma B de alto grado, probablemente de tipo Burkitt. Se inicia manejo de primera línea con quimioterapia con metrotexate, citarabina, y rituximab y corticoides a altas dosis, con mejoría parcial dada por el retiro de soportes con el primer ciclo; sin embargo, presenta recaída clínica con disfunción multiorgánica y asistolia que no responde a maniobras de reanimación con desenlace fatal.

\section{Discusión}

El linfoma de Burkitt es un subtipo de linfoma no Hodgkin cuya presentación a nivel testicular está relacionado con un pobre pronóstico, de hecho, debido a la ausencia de tejido linfoide en el testículo se cree que la mayoría de pacientes presenta diseminación sistémica al momento del diagnóstico o poco tiempo después. Existen diferentes factores de riesgo que pueden llevar al desarrollo de esa patología como lo son las inmunodeficiencias secundarias a drogas o infecciones y/o en pacientes trasplantados con un aumento del riesgo entre el $25 \%$ y el $50 \%$ que se eleva al $90 \%$ en pacientes $\mathrm{VIH}$ positivo en comparación con la población en general. Existe también asociación con enfermedades congénitas como el síndrome de ataxia telangiectasia, Wiscott-Alridge, síndrome de Sjogren y patologías como la tiroiditis de Hashimoto y enfermedad celíaca. $^{2}$

Las formas de presentación del linfoma testicular no Hodgkin son: linfoma oculto, afectación testicular de un linfoma ya conocido, linfoma primario sin afectación de otros órganos, siendo esa la forma más rara de presentación. ${ }^{2}$ Clínicamente es caracterizado por orquialgia intermitente, que puede asociarse o no a una masa fija e hidrocele hasta en el $40 \%$ de los casos. El compromiso de los dos testículos se presenta hasta en el 5\% de los casos; la afectación del testículo contralateral puede darse inclusive meses o años después de la orquiectomía radical y comportarse con histología diferente y puede asociarse a síntomas sistémicos como diaforesis, fiebre, anorexia y pérdida de peso entre el $25 \%$ al $41 \%$ de los pacientes. ${ }^{6}$ En estudios retrospectivos, se ha demostrado que a los 7,6 años, más de la mitad de los pacientes tienen recaída y aproximadamente el $40 \%$ tienen recurrencia extranodal. ${ }^{1}$ Histológicamente, la mitad de los linfomas extranodales son del patrón difuso de células $B$ gigantes, con invasión predominantemente del sistema nervioso central (SNC) y otros sitios como hueso, testículo e hígado, mientras que en el compromiso gastrointestinal se pueden observar diversos patrones histológicos que incluyen el linfoma Burkitt. En los niños, el subtipo histológico de linfoma testicular primario más frecuente es el de células foliculares, con compromiso secundario en los subtipos linfoblásticos. ${ }^{4,7}$

El estudio inicial puede realizarse con ecografía doppler testicular que puede demostrar áreas difusas o focales hipoecoicas con hipervascularidad asociado en el testículo, sin embargo, el estudio de extensión debe ser guiado con una tomografía axial computada de abdomen y/ o RM que permite una estatificación y un seguimiento adecuado. No obstante, la confirmación debe ser siempre histopatológica con el espécimen quirúrgico. El compromiso gastrointestinal a nivel terminal del intestino delgado, apéndice y el ciego son los sitios que se ven más comúnmente afectados en los linfomas no Hodgking incluyendo el subtipo de Burkitt, por ese motivo, las imágenes contrastadas van a permitir su identificación. ${ }^{8,9}$

El aspirado de médula ósea evalúa el compromiso medular, y la citometría y la citología de flujo han demostrado ser muy buenos predictores de compromiso del SNC, sin embargo, la RMN cerebral permite la caracterización y localización exacta de las lesiones. Por otro parte con la tomografía por emisión de positrones (PET- Scan), se logra una adecuada evaluación de posibles metástasis subclínicas no detectables en otros estudios imagenológicos. ${ }^{3}$

Al examen físico, la inspección de lesiones cutáneas y palpación testicular permite la identificación de compromiso extranodal en la región genital. Se deben solicitar pruebas de serología de VIH para el estudio de la inmunodeficiencia. Los marcadores tumorales convencionales como la alfafetoproteína (AFP) y la gonadotropina coriónica humana (HCG), no se elevan en ese tipo de tumores; no obstante, el lactato deshidrogenasa sérico (LDH) sí, y es indicador de agresividad y volumen 
tumoral, tomada en cuenta hoy en día para el diagnóstico y seguimiento. Con respecto a la inmunohistoquímica, la positividad de CD10, Bcl6 y MUM 1 han sido asociados al casi 90\% de los linfomas no Hodgkin, especialmente el difuso de células B gigantes. ${ }^{10,11}$

Hoy en día, el sistema de Ann Harbor, es usado para la clasificación y estatificación clínica del linfoma Hodking, ya que no fue diseñado específicamente para linfomas extranodales, sin embargo, fue sometido a una modificación para adaptarlo a los linfomas no Hodgkin ${ }^{3,11}$

Con respeto al tratamiento instaurado, el pilar del manejo y diagnóstico del linfoma testicular es la orquiectomía radical que permite un control local pero no curativo de la enfermedad, con reporte de sobrevida a 5 años del 16\% al $50 \%$. En la actualidad, se asocian manejos con quimioterapia y radioterapia local, e inclusive se están estudiando resultados con profilaxis y alternativas con anticuerpos monoclonales para evitar progresión y recaída al SNC; los resultados son variables dependiendo de los diferentes estudios documentados en la literatura. 5,12,13

Zucca y cols., demostraron de forma retrospectiva en 373 pacientes con linfoma testicular primario, un aumento en la sobrevida a 4,8 años y tiempo libre de progresión de la enfermedad de 4 años en pacientes con un índice de pronóstico internacional de linfoma no Hodgkin favorable, la ausencia de síntomas $B$, el uso de quimioterapia con antraciclina y radioterapia escrotal profiláctica. No obstante, aunque esos factores fueron significativamente favorables en la mejoría de sobrevida y disminución de recaída, presentan resultados desfavorables con respecto al compromiso del linfoma difuso de células B grande en otras localizaciones. ${ }^{1}$

Actualmente estudios adicionales han mostrado resultados diversos, encontrando en algunas series, que la asociación del rituximab al manejo con quimioterapia convencional con ciclofosfamida, doxirrubicun, vincristina y prednisona, no disminuía el riesgo de recaída ni progresión al SNC. No obstante, estudios recientes en pacientes con linfomas de células B agresivas de alto grado que presentan marcador $\mathrm{CD} 20+$, evidenciaron que la asociación del rituximab con ciclos de quimioterapia, demostró una mejoría tanto en la recaída y progresión al SNC, como también en la sobrevida. ${ }^{14,15}$

Por otro lado, la profilaxis intratecal y sistémica no demostró evidencia suficiente que evite el compromiso a nivel del sistema nervioso central, inclusive en paciente de alto riesgo $0^{7,11,16}$ Finalmente, el uso de la radioterapia a nivel escrotal, ha documentado no solo una disminución en la recaída del testículo contralateral, sino también en los ganglios regionales. ${ }^{17}$

\section{Conclusión}

El linfoma no Hodgkin testicular es una entidad rara que requiere de estratificación y manejo inmediato, el subtipo más frecuente es el linfoma difuso de células B grandes, sin embargo, se han reportado casos del subtipo de Burkitt con alta probabilidad de compromiso extranodal. Existen factores de riesgo que deben ser asociados con el desarrollo del linfoma no
Hodgkin, para permitir guiar el estudio y la estatificación pertinente.

El manejo de esa patología ha presentado múltiples modificaciones a través del tiempo, con el advenimiento de terapias combinadas y de profilaxis que permiten aumentar la sobrevida y disminuir el riesgo de recaída y progresión al SNC.

\section{Responsabilidades Éticas}

Protección de personas y animales. Los autores declaran que para esta investigación no se han realizados experimentos en seres humanos ni en animales.

Confidencialidad de los datos. Los autores declaran que han seguido los protocolos de su centro de trabajo sobre la publicación de datos de pacientes.

Derecho a la privacidad y consentimiento informado. Los autores declaran que en este artículo no aparecen datos de pacientes.

Conflicto de Intereses

No existe conflicto de intereses

Sitio donde se realizó el trabajo

Servicio de Urología- Hospital Universitario Mayor, Bogotá. Colombia

\section{Bibliografía}

1 Zucca E, Conconi A, Mughal TI, et al; International Extranodal Lymphoma Study Group. Patterns of outcome and prognostic factors in primary large-cell lymphoma of the testis in a survey by the International Extranodal Lymphoma Study Group. J Clin Oncol 2003;21(01):20-27

2 García Peñalver C, Lecki A, Sevilla Cecilia C, Díaz Delgado M, Parra Escobar JL, Sánchez Blasco E. Primary testicular lymphoma with extranodal involvement. Arch Esp Urol 2009;62(06):489-493

3 Vitolo U, Ferreri AJM, Zucca E. Primary testicular lymphoma. Crit Rev Oncol Hematol 2008;65(02):183-189

4 Zucca E. Extranodal lymphoma: a reappraisal. Ann Oncol 2008;19 (Suppl 4):iv77-iv80

5 Sasai K, Yamabe H, Tsutsui K, et al. Primary testicular nonHodgkin's lymphoma: a clinical study and review of the literature. Am J Clin Oncol 1997;20(01):59-62

6 Horne MJ, Adeniran AJ. Primary diffuse large B-cell lymphoma of the testis. Arch Pathol Lab Med 2011;135(10):1363-1367

7 Mihaljevic B, Vukovic V, Smiljanic M, et al. Single-center experience in the treatment of primary testicular lymphoma. Oncol Res Treat 2014;37(05):239-242

8 Satishchandra H, Sridhar AS, Pooja BP. Imaging of Burkitt's lymphoma-abdominal manifestations. J Cancer Res Ther 2013;9 (01):128-130

9 Cakiroglu B, Eyyupoglu SE, Dogan AN, Noseri U, Aksoy SH, Ozturk AB. Primary testicular lymphoma: Two case reports and review of the literature. Arch Ital Urol Androl 2016;87(04):332-334

10 Bhatia K, Vaid AK, Gupta S, Doval DC, Talwar V. Primary testicular non-Hodgkin's lymphoma-a review article. Sao Paulo Med J 2007; 125(05):286-288

11 Cheah CY, Wirth A, Seymour JF. Primary testicular lymphoma. Blood 2014;123(04):486-493

12 Linassier C, Desablens B, Lefrancq T, et al; GOELAMS Study Group. Stage I-IIE primary non-Hodgkin's lymphoma of the testis: results of a prospective trial by the GOELAMS Study Group. Clin Lymphoma 2002;3(03):167-172 
13 Wang C, Wang H, Wang Q, Shi B. Primary testicular lymphoma: experience with 13 cases and literature review. Int J Hematol 2013;97(02):240-245

14 Zouhair A, Herrmann E, Ugurluer G, Gaye PM, Mirimanoff RO, Ozsahin M. Primary testicular lymphoma. Swiss Med Wkly 2010; 140:w13076

15 Pfreundschuh M, Schubert J, Ziepert M, et al; German HighGrade Non-Hodgkin Lymphoma Study Group (DSHNHL). Six versus eight cycles of bi-weekly CHOP-14 with or without rituximab in elderly patients with aggressive CD20+ B-cell lymphomas: a randomised controlled trial (RICOVER-60). Lancet Oncol 2008;9(02):105-116

16 Fletcher CD, Kahl BS. Central nervous system involvement in diffuse large B-cell lymphoma: an analysis of risks and prevention strategies in the post-rituximab era. Leuk Lymphoma 2014;55(10):2228-2240

17 Ahmad SS, Idris SF, Follows GA, Williams MV. Primary testicular lymphoma. Clin Oncol (R Coll Radiol) 2012;24(05):358-365 